\title{
EFFECT OF SOLVENTS ON PARTICLE STRUCTURE, MORPHOLOGY AND OPTICAL PROPERTIES OF ZINC OXIDE NANOPARTICLES
}

\author{
A.Vanaja ${ }^{1}$ and K.Srinivasa $\mathrm{Rao}^{2}$ \\ ${ }^{1}$ Department of Physics, Lingayya's University, Old Faridabad, Haryana, India. \\ vanajatunuguntla@yahoo.com \\ ${ }^{2}$ Department of EIE. Lakireddy Balireddy College of Engg, Mylavaram,A.P., India \\ srinivasakarumuriegmail.com
}

\begin{abstract}
Nanostructured Zinc oxide Nanoparticles were synthesized by simple and low cost sol-gel process in aqueous medium. Zinc Nitrate, Potassium Hydroxide and Sodium hydroxide were used as precursors. The synthesized particles were further characterized by X Ray Diffraction (XRD), Scanning Electron Microscopy (SEM), Photo Luminescence (PL) and Optical Absorption Spectroscopy. The structure, morphology and optical properties are analyzed .XRD and SEM confirmed the formation of $\mathrm{ZnO}$ particles with average crystallite size of $36.89 \mathrm{~nm}$ and $21.59 \mathrm{~nm}$ obtained from solvents $\mathrm{NaOH}$ and $\mathrm{KOH}$. The emission peaks were detected by PL spectrum. The optical absorption spectra of the ZnO nanopowders showed blue-shift in wavelength corresponding to bulk. From the analyses of results, it is very clear that precursors used in the present study have played a vital role in surface morphology, structural and optical properties of $\mathrm{ZnO}$ nanoparticles.
\end{abstract}

\section{KEYWORDS:}

ZnO nanoparticles, UV-Vis optical absorption, SEM, X Ray Diffraction.

\section{INTRODUCTION}

Nanotechnology is a field of science and technology of controlling matter on a scale between 1100 nanometers. It is a highly multidisciplinary field, bringing together many fields, including electrical and mechanical engineering, physics, chemistry, and biosciences. In fact, one nanometer $(\mathrm{nm})$ is one billionth of a meter, tens of thousands times smaller than the width of a human hair. Nanomaterials have been important in the materials field for a long time due to their unusual mechanical, electrical, optical and magnetic properties. Semiconductor nanoparticles (NPs) are a very important topic in the ongoing research activity across the world. During the past decade a considerable effort has been spent in the preparation and investigation of the family of II-VI nanoscale semiconductors as they exhibit size-dependant properties like scaling of the energy gap and corresponding change in the optical properties.

Among all the II-VI compounds, Zinc oxide $(\mathrm{ZnO})$ is attracting tremendous attention due to its interesting properties like wide direct band gap of $3.3 \mathrm{eV}$ at room temperature and high exciton binding energy of $60 \mathrm{meV}$ ( Shriwas Ashtaputre et al., 2005) . $\mathrm{ZnO}$ is widely used in a number of applications like varistors (Rana etal., 2010) UV lasers, gas sensors, photo printing, 
electrochemical nanodevice, sunscreen lotion cosmetics and medicated creams (Ravichandrika., et al 2012) due to its several properties such as good transparency, high electron mobility, strong room temperature luminescence .Different types of techniques have been used to synthesize $\mathrm{ZnO}$ such as sputtering, spray pyrolysis (Abdelkader Djelloul et al.,2008) Solvothermal (Wang et al., 2005) Hydrothermal (Ni YH et al., 2005) ,Ball milling method (Lemine et al, 2010) and Wet Chemical method (Satyarana et al 2012; Shah and Alshahry,2009; Singh et al,2012) .Sol-gel method was reported as one of the well known procedures for the deposition of metal oxide nanoparticles due to its many advantages of being non vacuum, low substrate temperature deposition and low cost.

In the present study $\mathrm{ZnO}$ nanoparticles were synthesized by a sol-gel technique from Zinc Nitrate, Potassium Hydroxide and Sodium Hydroxide in aqueous medium. The structure, morphology and optical properties of $\mathrm{ZnO}$ were investigated by XRD, SEM, UV-VIS and PL measurements.

\section{EXPERIMENT DETAILS}

\subsection{Materials}

Zinc Nitrate, Sodium Hydroxide $(\mathrm{NaOH})$, Potassium Hydroxide $(\mathrm{KOH})$ were purchased and used without further purification.

\subsection{Synthesis of Zinc Oxide Nanoparticles}

The zinc oxide $(\mathrm{ZnO})$ nanoparticles were prepared by sol-gel method using zinc nitrate and sodium hydroxide as precursors In this experiment Zinc nitrate $(0.4 \mathrm{~mol})$, was dissolved in the distilled water. Then the solution was kept under constant stirring using magnetic stirrer to completely dissolve the zinc nitrate for one hour. After complete dissolution of zinc nitrate, 0.8 mol of sodium hydroxide solution was added under constant stirring, drop by drop touching the walls of the vessel. The reaction was allowed to proceed for $2 \mathrm{~h}$ after complete addition of sodium hydroxide. After the completion of reaction, the solution was allowed to settle for overnight and the supernatant solution was then discarded carefully. The remaining solution was centrifuged for $10 \mathrm{~min}$ and the supernatant was discarded. Thus obtained nanoparticles were washed three times using distilled water. Washing was carried out to remove the byproducts that were bound with the nanoparticles. After washing, the nanoparticles were dried in micro oven at a maximum temperature of $70^{\circ} \mathrm{C}$ for several hours. During drying, complete conversion of zinc hydroxide in to zinc oxide takes place.

In a similar manner, $\mathrm{ZnO}$ nanoparticles were synthesized from zinc nitrate with potassium hydroxide. Further, the two samples of $\mathrm{ZnO}$ nanoparticles were characterized by for their optical and nano-structural properties using X-ray diffraction, scanning electron microscopy, UV-vis optical absorption and Photoluminescence spectra.

\section{RESULTS AND DISCUSSION}

\subsection{Ray Diffraction}

$\mathrm{ZnO}$ structures with different grain sizes can be obtained by using different precursors. Fig1.1 and 1.2 represent the $\mathrm{X}$-ray diffraction patterns of $\mathrm{ZnO}$ nanoparticles prepared with different precursors $\mathrm{NaOH}$ and $\mathrm{KOH}$.From the patterns it is evident that a definite line broadening of the XRD peaks indicate nano level range of particles in the the prepared material. From XRD patterns analyses, we determined peak intensity, position and width, full-width at half-maximum 
(FWHM) data. In Fig 1.1 the diffraction peaks are $31.940^{\circ}, 34.612^{\circ}, 36.433^{\circ}, 47.692^{\circ} .56 .736^{\circ}$ $, 62.987^{\circ}, 66.51^{\circ}, 68.055^{\circ}, 69.116^{\circ}, 72.656^{\circ}, 77.02^{\circ}, 81.41^{\circ}$ and $89.667^{\circ}$ correspond to lattice planes (100), (002 ), (101), , (102),,(110) ,(103), (200), (112), ,(201) ,(004), (202) , ) respectively. In the Fig 1.2 the diffraction peaks are at $31.946^{\circ}, 34.631^{\circ}, 36.448^{\circ}, 47.755^{\circ}$ $, 56.769^{\circ}, 62.928^{\circ}, 66.45^{\circ}, 68.09^{\circ}, 69.19^{\circ}, 77.17^{\circ}$ and $81.56^{\circ}$ correspond to lattice planes (100) ,(002),,(101),,(102) (110),(103),(200),,(112),(201),(004), (202),(104) respectively.

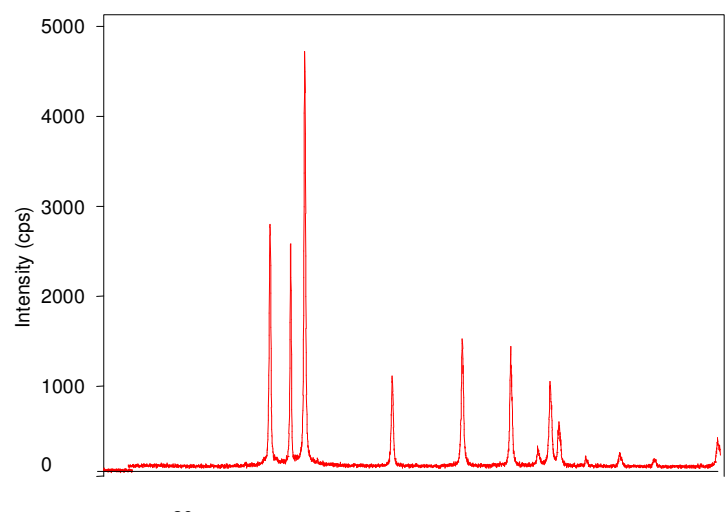

20

40

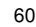

80

2-theta (deg)

Figure: 2.1

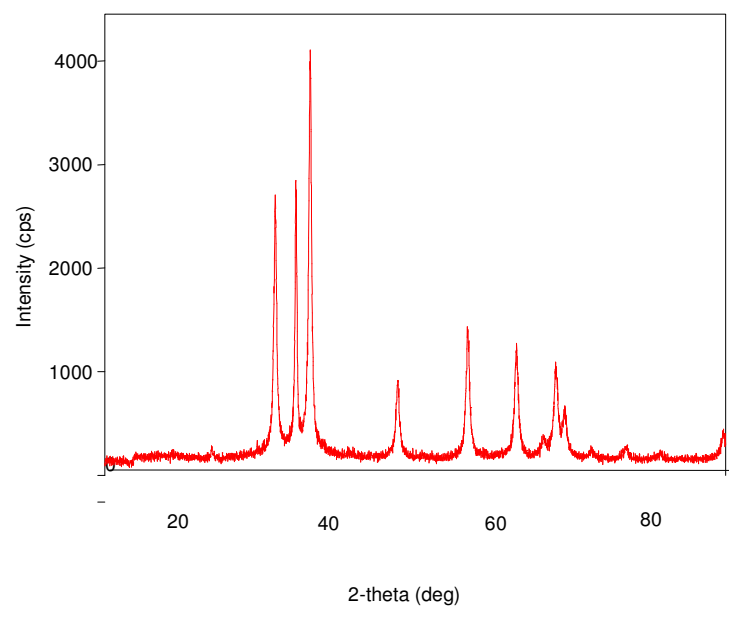

Figure: 2.2

Figure 1.1: XRD Spectrum of $\mathrm{ZnO}$ nanoparticles synthesized using $\mathrm{NaOH}$. Figure 1.1: XRD Spectrum of $\mathrm{ZnO}$ nanoparticles synthesized using $\mathrm{KOH}$,

The diffraction peaks can be indexed for wurtzite type hexagonal structure of $\mathrm{ZnO}$. No peaks from other phase of $\mathrm{ZnO}$ or impurities are observed suggesting that high-purity $\mathrm{ZnO}$ phase was obtained. From the diffraction patterns it is confirmed that the plane (101) is more intense than 
other peaks. The average crystalline size of the particles is calculated using Debye Scherer formula,

$$
d \text { avg }=0.9 \lambda / \beta \operatorname{Cos} \theta .
$$

Where

$$
\begin{aligned}
\text { davg } & =\text { Average crystaline size } \\
\lambda & =\text { Wavelength of incident beam }(1.5406 \AA), \\
\beta & =\text { FWHM in radians, } \\
\theta & =\text { Scattering angle in degree }
\end{aligned}
$$

The average crystallite size of the samples is found to be $36.89 \mathrm{~nm}$ in the case of $\mathrm{ZnO}$ with $\mathrm{NaOH}$ while in the case of $\mathrm{ZnO}$ with $\mathrm{KOH}$ is $21.59 \mathrm{~nm}$. The XRD pattern for $\mathrm{ZnO}$ nanoparticles obtained with water medium with solvents $\mathrm{NaOH}$ and $\mathrm{KOH}$ shows much sharper peaks. This shows the crystallinity is more synthesized $\mathrm{ZnO}$ nanopowders.

\subsection{Scanning Electron Microscopy}

The morphology and size of the synthesized $\mathrm{ZnO}$ particles were studied by scanning electron microscopy (SEM). Fig 2.1 and Fig 2.2 represent the effect of solvents $(\mathrm{NaOH}$ or $\mathrm{KOH}$ ) on particle morphology of $\mathrm{ZnO}$ Nanopowders observed at different magnifications. These pictures confirm the formation of $\mathrm{ZnO}$ nanoparticels SEM micrographs of $\mathrm{ZnO}$ nano powders prepared with $\mathrm{NaOH}, \mathrm{KOH}$ found to have an irregular spherical shape (whose size in the range of nanometers) along with narrow particle size distribution. It is also observed that most of the particles exhibit some faceting. The estimated particle size from SEM pictures found to be varying between $17 \mathrm{~nm}$ and $25 \mathrm{~nm}$ for the sample synthesized with $\mathrm{KOH} 30-50 \mathrm{~nm}$ for the sample synthesized from $\mathrm{NaOH}$. The morphology observed in the samples show fine grains of $\mathrm{ZnO}$ converted in to particles when observed at different magnifications. Influence of $\mathrm{KOH}$ tends to cause the particle size to decrease, which can be attributed to the decrease in solubility.

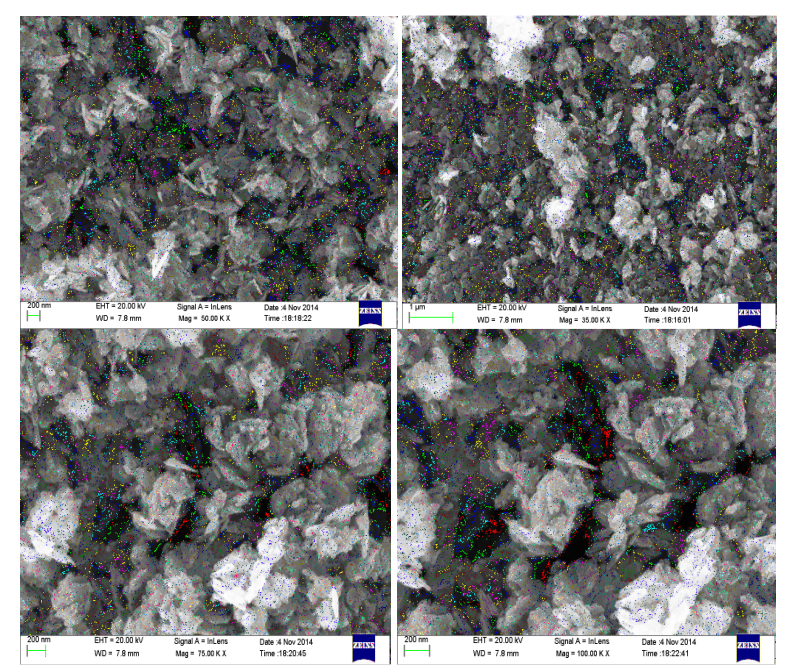

Figure: 2.1 


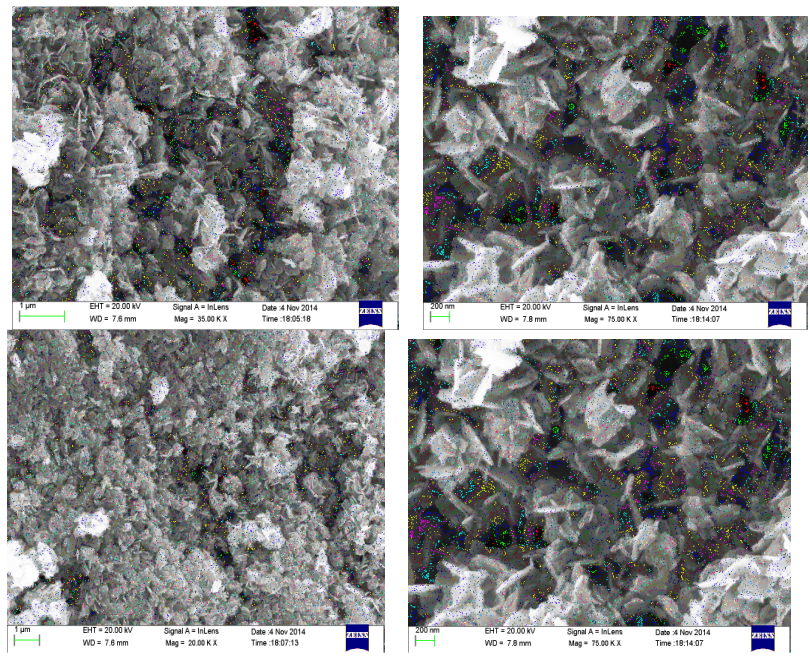

Figure: 2.2

Fig 2.1: SEM images of sample at different magnifications obtained using solvent $\mathrm{NaOH}$ Fig 2.2: SEM images of the sample at different magnifications obtained using solvent $\mathrm{KOH}$.

\subsection{UV-Visible Absorption Spectra}

Absorption spectroscopy is a powerful non-destructive technique for exploring the optical properties of semiconducting nanoparticles. The optical study was performed to evaluate the potentially useful optical qualities of the NPs . Fig 3.1 and Fig 3.2 represent UV-Vis absorption spectra of $\mathrm{ZnO}$ nanoparticles synthesized using $\mathrm{NaOH}$ and $\mathrm{KOH}$ respectively. From the spectra, it is very clear that both the samples exhibit strong absorption band with maxima around 345-400 $\mathrm{nm}$ and another excitonic absorption band located in the range $200-250 \mathrm{~nm}$

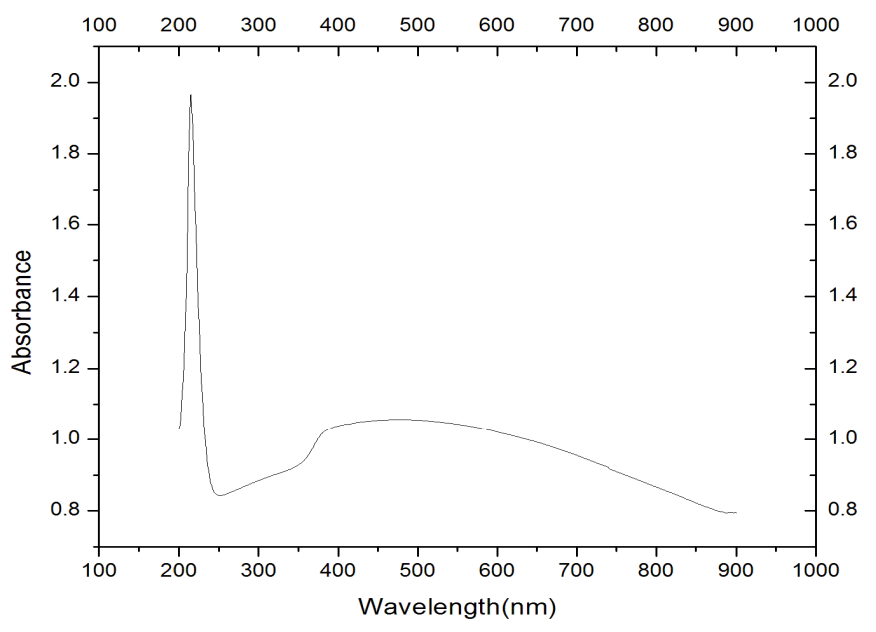

Figure 3.1 


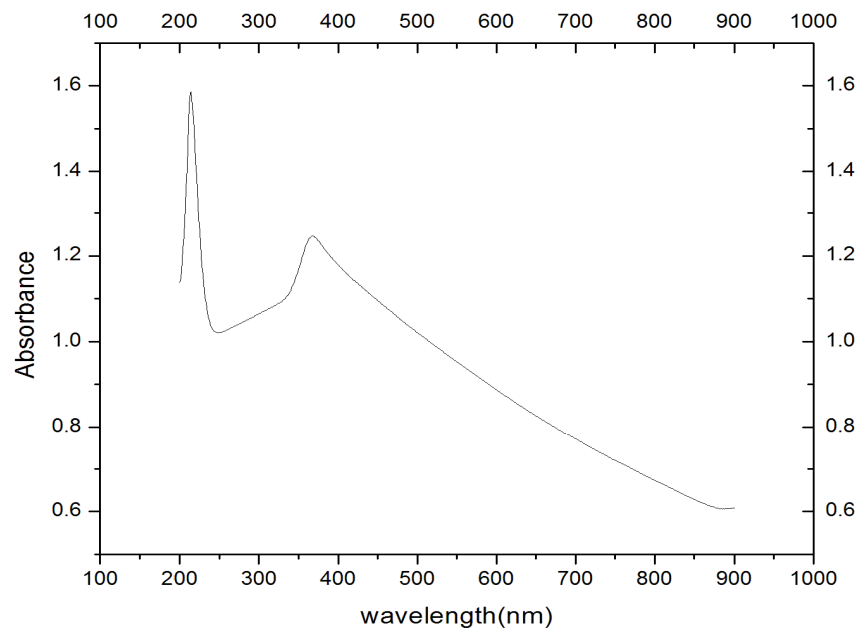

Figure 3.2

Fig3.1: Optical absorption Spectra of $\mathrm{ZnO}$ NPs synthesized using $\mathrm{NaOH}$ Fig3.2: Optical absorption Spectra of ZnO NPs synthesized using $\mathrm{KOH}$

The value of optical band gap (Eg) has been estimated by fitting the rising end and plateau of the absorption spectra to the sigmoidal formula developed by O' Donnell and coworkers. The band gap corresponding to strong absorption is given by $0.95 \mathrm{eV}$. Which is higher than bulk $\mathrm{ZnO}$ material. The blue shift in the excitonic absorption clearly indicates the quantum confinement property of NPs. It can be seen from the spectra that solvents used do not have any considerable impact on the position of strong absorption band so that size of the particle. But there is very small shift in the absorbance maximum with the use of precursors in order $\mathrm{NaOH}, \mathrm{KOH}$. The shift might be associated with the broader nanoparticle distribution such that broader exciton absorbance.

\subsection{Photoluminescence Spectra}

Photoluminescence is a powerful tool for providing essential information about the physical properties of materials at molecular levels, including shallow and deep level defects and band gap state for energy level. Fig 3.1 and fig 3.2 represent the PL Spectra of ZnO NPs synthesized using $\mathrm{NaOH}$ and $\mathrm{KOH}$ respectively. The PL spectra in both the samples exhibit two emission peaks, one is located at around $550 \mathrm{~nm}$ corresponds to the near band gap excitonic emission and the other is located at around $600 \mathrm{~nm}$. Both the samples showed the prominent peak around $600 \mathrm{~nm}$ corresponds to the blue green emission. These emissions were found to be extremely broad and this broadening may be due to phonon assisted by transition. As observed in UV-visible optical absorption spectra, there is no significant change in the position of both the emission peaks. Due to broader distribution of nanoparticles in $\mathrm{NaOH}$, the intensity and full-width at half-maximum (FWHM) are found to be small in comparison with the $\mathrm{ZnO}$ nanoparticles in the presence of $\mathrm{KOH}$. 


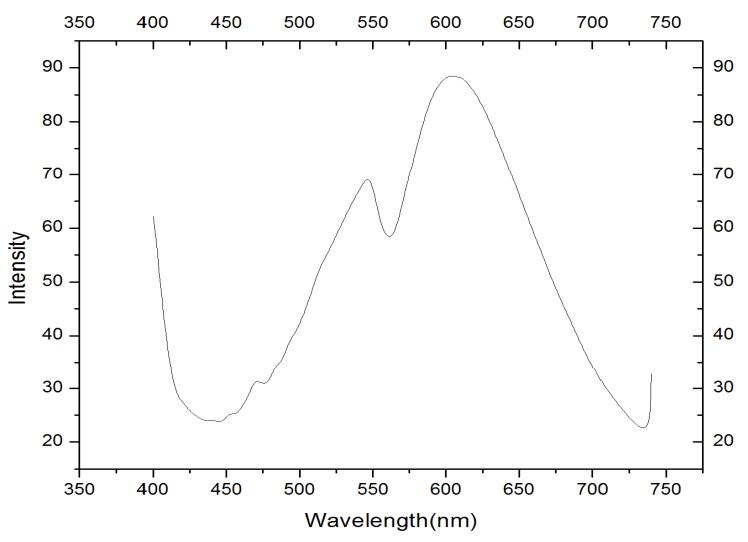

Figure 3.1

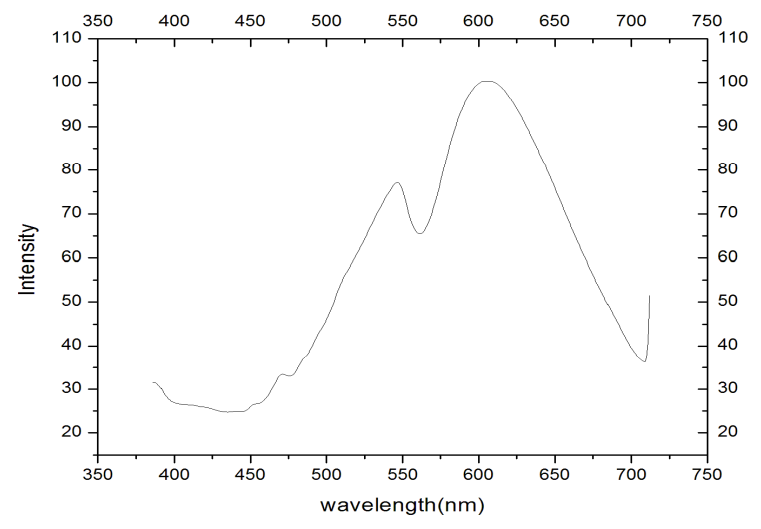

Figure 3.2

Fig 3.1: PL spectra of $\mathrm{ZnO}$ NPs synthesized using $\mathrm{NaOH}$ Fig 3.2: PL spectra of $\mathrm{ZnO}$ NPs synthesized using $\mathrm{KOH}$

\section{CONCLUSION}

$\mathrm{ZnO}$ nanoparticles were synthesized using simple and cost effective sol-gel process $\mathrm{n}$ the presence of $\mathrm{NaOH}$ and $\mathrm{KOH}$ in aqueous medium. The effect of solvents on structure, morphology and optical properties of $\mathrm{ZnO}$ nanopowders were analyzed using XRD, SEM, Optical absorption and Photo Luminescence. The XRD analysis demonstrates that the nanoparticles have the hexagonal wurtzite structure.SEM confirms the morphology and size of the nanoparticles. The decrease in the particle size is observed due to the influence of $\mathrm{KOH}$. Both the samples exhibit bands in the same range but samples with $\mathrm{NaOH}$ found to exhibit broader distribution both the samples showed the prominent peak around $600 \mathrm{~nm}$ corresponds to the blue green emission of PL spectra. The sol-gel route employed in this work is simple, cost effective and free of pollution and therefore, the technique can be extended to prepare many other important semiconducting metal oxide nanoparticles. These $\mathrm{ZnO}$ nanoparticles can be used in different industrial applications viz., luminescent material for fluorescent tubes, active medium for lasers, sensors etc. From the 
International Journal of Advances in Materials Science and Engineering (IJAMSE) Vol.4, No.2, April 2015

analyses of results, it is very clear that precursors used in the present study have played a vital role in surface morphology, structural and optical properties of $\mathrm{ZnO}$ nanoparticles.

\section{References}

[1] K. SURESH BABU and V. NARAYANAN(2013), Chem Sci Trans., 2(S1), S33-S36

[2] B. Srinivasa rao, B. Rajesh kumar, V. Rajagopal reddy, T. Subba rao(2011), Chalcogenide Letters, 8 (177). 